\title{
LA PROMOCIÓN DE UNA ECONOMÍA NO-CAPITALISTA: EL GRUPO MONDRAGÓN*
}

Resumen: Mondragón es hoy el séptimo grupo empresarial de España según la variable cifra de "ventas", y el tercero de acuerdo con la variable "empleo". Se trata de una cooperativa que ofrece una alternativa no capitalista cuyo éxito demuestra la posibilidad de transformación social, dejando obsoletas las Teorías de la Reproducción que defienden que la educación no puede contribuir a superar las desigualdades sociales. Este artículo analiza la relevancia que Mondragón ha otorgado a la educación a lo largo de más de 50 años, convirtiéndola en un elemento clave para su éxito y para la mejora de las condiciones de vida de las personas que participan en esta experiencia.

Palabras clave: reproducción, transformación, éxito empresarial, educación, cooperativismo, economía social.

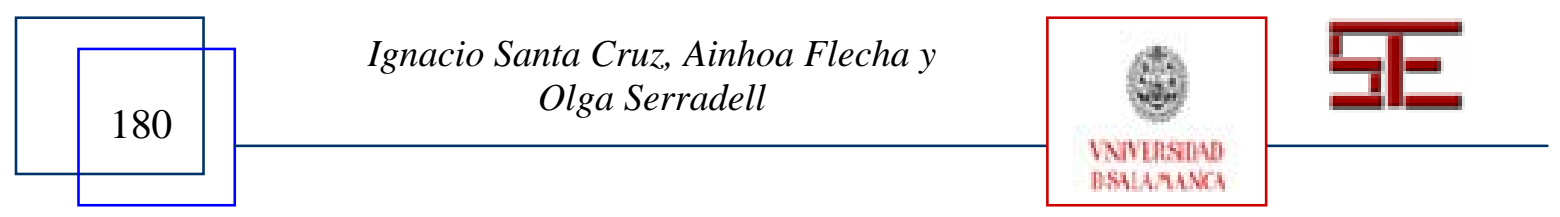




\begin{tabular}{c|c} 
Revista Electrónica Teoría de la Educación. \\
Educación y Cultura en la Sociedad de la Información.
\end{tabular}

\section{THE PROMOTION OF A NON-CAPITALIST ECONOMY: THE MON- DRAGÓN GROUP}

Abstract: Mondragón is at present the seventh entrepreneurial group in Spain according to sales volume and the third according to employment. As a cooperative, it offers a non-capitalist alternative of success, demonstrating that social transformation is possible and invalidating the theories of reproduction, which defend that education can not contribute to the overcoming of social inequalities. The present article analyses the importance given to education by Mondragón throughout its 50 years of history, making out of it a key factor both for the corporation's success as well as for the improvement of the life condition of those who participate in this experience.

Keywords: reproduction, transformation, business success, education, cooperativism, social economy.

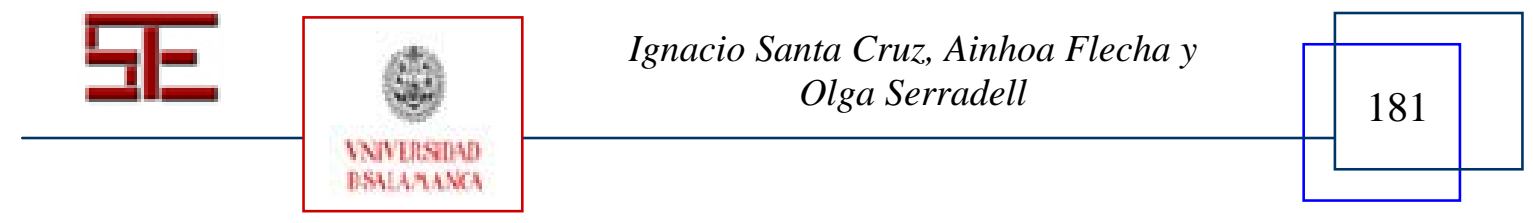




\section{LA PROMOCIÓN DE UNA ECONOMÍA NO-CAPITALISTA: EL GRUPO MONDRAGÓN}

Ignacio Santa Cruz.

inaki.santacruz@uab.cat

Ainhoa Flecha.

Ainhoa.Flecha@uab.cat

Olga Serradell.

Olga.Serradell@uab.cat

Universitat Autònoma de Barcelona.

\section{1.- INTRODUCCIÓN: EL ORIGEN DE UNA UTOPÍA REAL}

Mondragón es hoy el primer grupo empresarial del País Vasco. Integrado por 264 empresas y entidades, es el séptimo grupo empresarial de España según la variable cifra de "ventas" y el tercero de acuerdo con la variable "empleo". Aunque cooperativas hay muchas, la singularidad de Mondragón recae precisamente en su éxito erigiéndose en un claro ejemplo de empresa que consigue triunfar en un mundo capitalista a través de principios no capitalistas. En un momento en que se están cuestionando las bases de nuestro sistema económico, es fundamental que la sociología presente modelos alternativos que pueden inspirar un nuevo orden internacional. Mondragón compite en los mercados internacionales utilizando métodos de organización democráticos y entre sus objetivos se encuentran la creación de empleo, la promoción humana y profesional de sus trabajadores y el compromiso de desarrollo con su entorno social.

El análisis de la experiencia de Mondragón refleja como a partir de la participación, la democracia, la solidaridad y otros valores no capitalistas se puede llegar a un modelo de gestión eficiente que aporte beneficios tanto a trabajadoras y trabajadores como a su entorno social. Entre los elementos que hacen posible un desarrollo tan positivo se encuentra la importancia otorgada a la educación en las diferentes áreas de Mondragón. La formación es considerada una herramienta básica del Área de Finanzas, durante el 2007 el 90\% de su plantilla total realizó alguna actividad formativa. Por esa razón, en 2007 se aplicó un 17\% más que en 2006 del Fondo de Educación y Promoción de Caja Laboral, un total de 12,6 millones de euros, para la promoción de diferentes actividades, entre

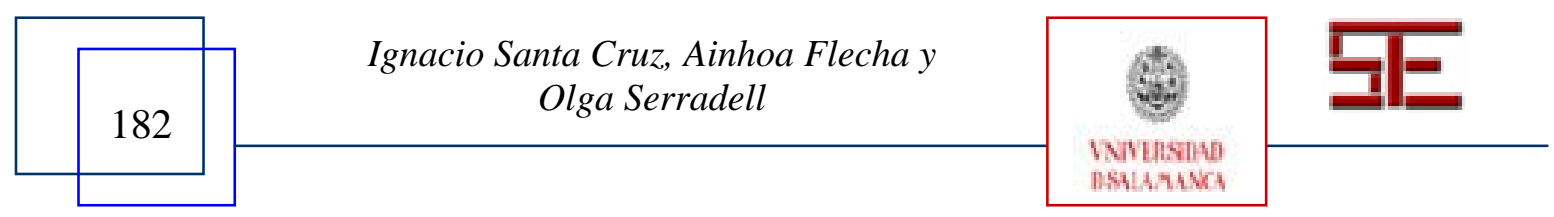




\begin{tabular}{c|c} 
Revista Electrónica Teoría de la Educación. \\
Educación y Cultura en la Sociedad de la Información.
\end{tabular}

ellas la formación (Mondragón, 2007 11). En el área de industria la cantidad dedicada a formación fueron 9 millones de euros (Mondragón, 2007 20). Como muestra el siguiente gráfico, la educación ocupa un lugar central en la organización:

Gráfico 1: Modelo de Gestión Corporativo. Principios Básicos Corporativos

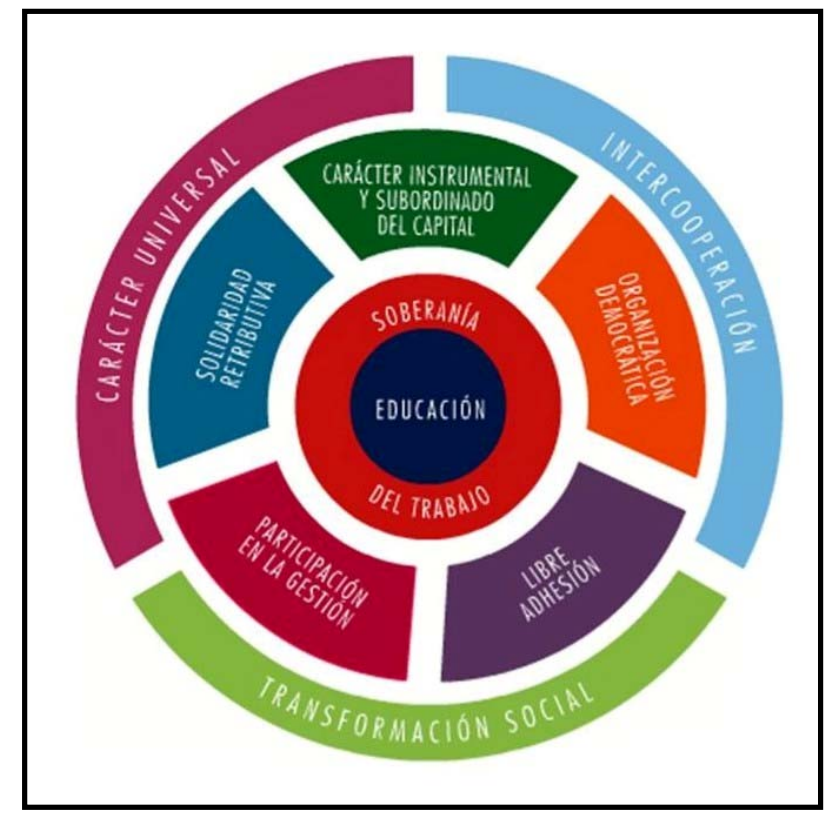

Fuente: http://www.mcc.es/esp/quienessomos/modelo/index.html.

Es imposible explicar el desarrollo de la experiencia de Mondragón sin tener en cuenta el trabajo realizado por D. José María Arizmendiarreta desde que en 1941 llegó a la parroquia de Mondragón. Su prioridad fue siempre mejorar las condiciones de vida de la población ante las consecuencias de la guerra. Para ello, entre las diferentes actividades que puso en marcha con los jóvenes destacan las orientadas a la formación. D. José María fundó la Escuela Profesional de Mondragón, en la que era profesor. Esta escuela impartía una educación teórica muy orientada a la aplicación práctica. Así, en 1955, este joven profesor y sacerdote creó junto con 5 alumnos los Talleres Ulgor (actualmente Fagor Electrodomésticos), al mismo tiempo que se impulsaban otros proyectos cooperativos desde la Escuela Profesional. Por ejemplo, Lagun-Aro (1959) cubría las prestaciones de la Seguridad Social a las que los cooperativistas no accedían por ser propietarios; Eroski fue producto de la fusión de nueve pequeñas cooperativas locales en 1969, y en la actualidad es una de las principales empresas del grupo con un total de 50.600 empleos; Caja Laboral fue creada en 1959 para captar el ahorro popular y obtener recursos para el desarrollo cooperativo. Cabe señalar que el apoyo que proporcionó esta entidad a las nuevas cooperativas que se creaban fue decisivo, por la función financiera que tenía la entidad pero también por el rol de asesoramiento que adquirió.

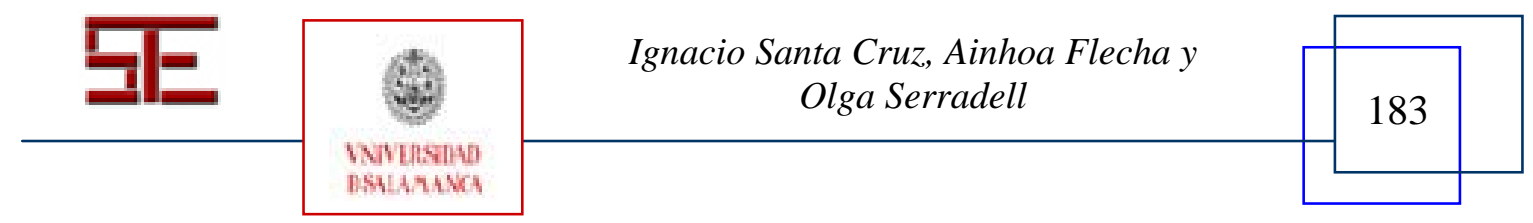


Los orígenes de Mondragón aparecen fuertemente vinculados a la formación, tanto académica como profesional. El sólido tejido educativo de Mondragón ha tenido y tiene un papel determinante en el desarrollo de la experiencia y en su éxito empresarial en el mundo. A pesar de la importancia que tiene el contexto económico en el desarrollo de cualquier experiencia empresarial, la educación ha sido y sigue siendo en la actualidad un factor clave. Mondragón cuenta hoy con varios Centros de Formación Profesional y una Universidad propia. En su creación en 1962, la Eskola Politeknikoa ya formaba a más de 1.000 estudiantes en las actividades desarrolladas en las cooperativas, pero hasta 1969 no fue reconocida oficialmente como Escuela de Ingeniería Técnica Industrial. En 1997 se fusionó con otras cooperativas para crear Mondragón Unibertsitatea (MU en adelante), que en la actualidad supera los 4.000 estudiantes. Esta inversión en formación iba unida desde el inicio a una idea de garantizar la igualdad de oportunidades y, de hecho, con ese fin se creó Alecop en 1966, una cooperativa que daba a los alumnos un trabajo que les permitía compatibilizar estudio y vida laboral.

En el curso 2006-2007 la MU tenía 3.327 alumnos matriculados en las 25 titulaciones de grado que ofrece esta universidad y 357 alumnos en las de postgrado (Mondragón, 2007 43). La formación académica que ofrece esta universidad cubre tres ámbitos: Técnico, de Gestión y de Educación y Humanidades. La Escuela Politécnica Superior imparte en la actualidad hasta 11 titulaciones de Ingeniería, la diplomatura y licenciatura en Ciencias Empresariales y Secretariado de Dirección, 6 titulaciones de magisterio, la licenciatura en Psicopedagogía y la de Comunicación Audiovisual. Además, MU imparte dobles diplomaturas en Ingeniería Industrial con universidades francesas de Toulouse (École Central de Nantes, INSA y ENSEEIHT).

Pero esta inversión en formación destacó también en la etapa comprendida entre 1970 y 1990, con la creación de Otalora, un centro de formación continua de directivos y miembros de los órganos sociales de la Corporación en los ámbitos empresarial y cooperativo, e Ikerlan, un centro de investigación de las innovaciones tecnológicas y desarrollo de sus aplicaciones en la industria. Para ello contaba con el apoyo y contribuciones de Caja Laboral y Mondragón Eskola Politeknikoa. En 2007 Otalora organizó actividades de formación en el área de Desarrollo Directivo en las que participaron hasta 695 personas. En los programas Básicos Generales hubo 497 asistentes y hasta 198 en los programas específicos y seminarios cortos de contenido práctico. En lo que se refiere a Ikerlan, donde hoy trabajan 202 investigadores técnicos y 37 becarios, obtuvo en 2007 6,55 millones de euros para proyectos de investigación genérica y estratégica financiados por la administración vasca, española y europea. A su vez destinó 11,02 millones a proyectos de I+D, incrementando el de 2006 (Mondragón, 2007, 40).

El grupo Mondragón tenía ya, en 1970, 40 cooperativas, impulsadas sobre todo por Talleres Ulgor y Caja Laboral, y hasta 1990 siguió con una dinámica de crecimiento de las ventas, de las cooperativas y del empleo. La profunda crisis que sufrió la economía española a finales de los años setenta afectó también a Mondragón, pero mientras algunas de las cooperativas del grupo obtenían por primera vez resultados negativos, otras, las que habían iniciado una política de exportación, pudieron resistir los efectos de la crisis.

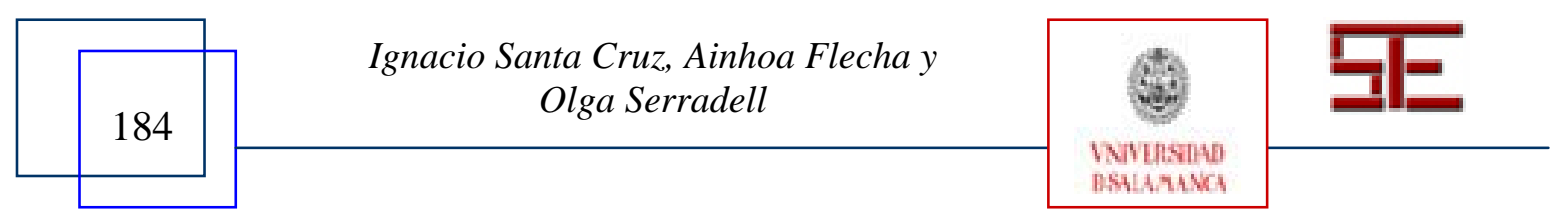




\begin{tabular}{c|c} 
Revista Electrónica Teoría de la Educación. \\
Educación y Cultura en la Sociedad de la Información.
\end{tabular}

Sus consecuencias fueron mucho más negativas para otras empresas que no se basaban en el modelo cooperativo de modo que, además de sobrevivir a la crisis, las cooperativas de Mondragón salieron reforzadas. Lo que permitió a Mondragón hacer frente a la crisis fueron los procedimientos que caracterizaban su modelo organizativo y empresarial, así como medidas específicas decididas por la Asamblea General en la que participan y votan todos los socios. A continuación destacamos cinco de los procedimientos y medidas que permitieron superar mejor la crisis que el resto del tejido empresarial español basado en un modelo no cooperativo:

1) La capitalización del $90 \%$ de los beneficios (tan sólo el 10\% sale de las cooperativas para Obra Social) permite el refuerzo permanente de los recursos.

2) Se habían creado diversos Grupos Comarcales inspirados en el Grupo Ularco y una parte de los beneficios obtenidos en unas cooperativas se reinvirtieron en las que más lo necesitaban.

3) Caja Laboral ofreció créditos a las cooperativas a intereses muy bajos y, en algunos casos, incluso sin ningún interés.

4) La política de reubicaciones permitió que los socios excedentarios en una cooperativa tuvieran preferencia y la obligación de ocupar, temporal o definitivamente, un puesto en otra cooperativa. Esta medida ajustaba los costes de personal sin que los trabajadores quedaran en paro, en un momento en que se empezaban a producir excedentes de plantilla en algunas de las cooperativas.

5) La política retributiva contemplaba dos pagas extraordinarias al año para los socios, que variarían en función de la situación económica y financiera de la cooperativa. Los socios realizaron un importante esfuerzo, ya que durante los años de crisis, en más de una ocasión se capitalizaron estas pagas para hacer frente a las dificultades de las cooperativas. En la actualidad se aplica para todas las cooperativas el Principio de Solidaridad Retributiva, que establece un marco solidario de la retribución al trabajo y del horario de trabajo anual. Tal como se indica en el informe anual de 2007:

Asimismo, la Política Retributiva contempla abanicos salariales para que no exista una amplia dispersión entre los salarios superiores e inferiores, y remuneraciones homologables con las de los trabajadores asalariados del entorno sectorial y territorial de las cooperativas. (Mondragón, 2008, 64).

Las medidas mencionadas fueron aprobadas por votación individual de los socios en la Asamblea General y, en su día, constituyeron la clave para superar la profunda crisis que vivía la economía española en aquel momento. Sin embargo, hay que destacar que estas medidas nunca se habrían podido llevar a cabo sin la actitud solidaria de los trabajadores y trabajadoras. Esta solidaridad, fruto de su fuerte identificación con la organi-

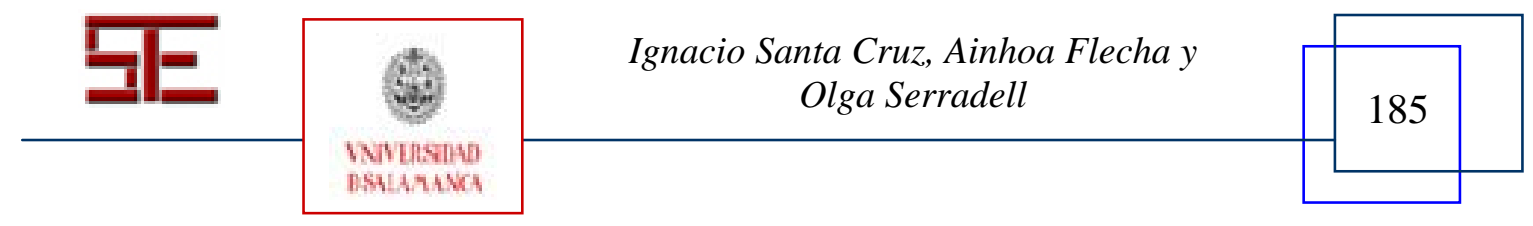


zación, les permitió priorizar el interés colectivo ante el suyo propio y superar la crisis a nivel individual y colectivo.

En la segunda mitad de los ochenta, junto a un nuevo escenario económico más favorable, Mondragón desarrolló cambios organizativos importantes en la política exportadora, incorporando empresas no cooperativas y constituyendo Grupos Comarcales. La entrada de España en la Comunidad Europea intensificó los debates sobre el futuro del grupo, sobre todo en lo que se refiere al mantenimiento de los valores cooperativos y dar respuesta a las nuevas exigencias del mercado y la sociedad. Caja Laboral desarrolló desde 1984 una actividad específicamente financiera con gran éxito, expandiendo el número de oficinas e instalándose fuera del País Vasco, en Madrid y Barcelona, mientras Eroski tomaba fuerza. Actualmente, y ante la actual situación económica mundial, cabe destacar los porcentajes sensiblemente superiores a la media del sector obtenidos por Caja Laboral, que concluyó el ejercicio de 2008 con un beneficio neto de 100,5 millones de euros y registró un incremento del 11,8\% de los recursos en balance y del 10,5\% en la concesión de créditos.

En 1984 se creó por primera vez una institución común al conjunto: el Congreso, el órgano que toma las decisiones y las lleva a cabo a través de una Comisión Permanente. Los principios básicos de Mondragón se definieron en los congresos de 1987 y 1989, recogiendo además de la doctrina de D. José María y su propia experiencia, los principios del cooperativismo universal. A partir de 1990 se produjeron cambios en el proceso organizativo y el Grupo se transformó en Corporación. En el Congreso Cooperativo de 1991, la organización de los negocios pasó a ser sectorial, en lugar de estar montada en base a la ubicación como hasta entonces. A partir de este momento se crearon tres grupos (industrial, financiero y de distribución) cuyo éxito se ha comprobado con el tiempo. Junto a su nueva expansión, el proyecto de Mondragón seguía reforzando el papel de la formación a través, por ejemplo, del desarrollo de actividades educativas como Eteo (formación en Administración y Dirección de Empresas), Irakasle Eskola (Escuela Universitaria de Profesorado), Eskola Politeknikoa Txorierri y Eskola Politeknikoa LeaArtibai. La formación se planteó como uno de los retos de los planes estratégicos elaborados para 1994-1996 y 1997-2000, junto a otros como la comunicación, el desarrollo, la internacionalización, la inversión o el empleo. Estos planes incluían aspectos que respondían más a las características de los diferentes mercados como una gestión orientada a las unidades de negocio o la incorporación de la satisfacción del cliente, el compromiso con el entorno y la satisfacción de socios y trabajadores. Muchos de estos retos fueron conseguidos. Más adelante se incluyó el área de Conocimiento en la organización de Mondragón, de modo que en la actualidad la forman cuatro áreas: Finanzas, Industria, Distribución y Conocimiento.

Como ya hemos comentado, en 1997 se creó Mondragón Unibertsitatea, una iniciativa promovida por Mondragón Eskola Politeknikoa, Irakasle Eskola y Eteo, y que proporcionó una gran autonomía para la formación de profesionales al proyecto de Mondragón. La creación de una universidad, así como otras de las acciones realizadas para responder a los retos planteados, respondían a los cuatro valores corporativos del pro-

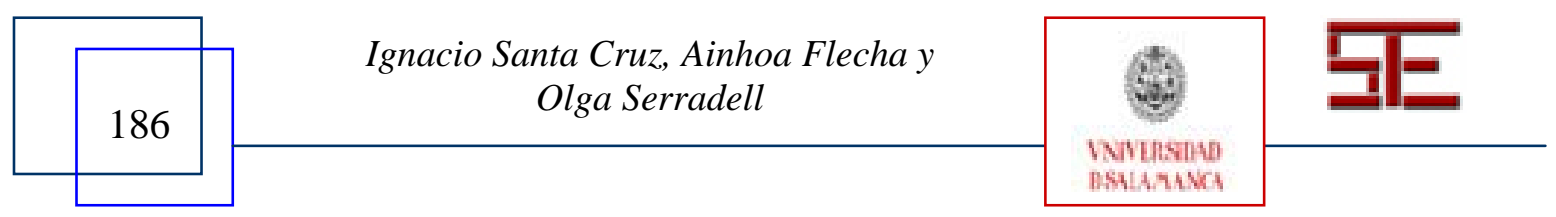




\begin{tabular}{c|c}
\hline 1 & $\begin{array}{c}\text { Revista Electrónica Teoría de la Educación. } \\
\text { Educación y Cultura en la Sociedad de la Información. }\end{array}$ \\
\hline & TESI, 10(3), 2009, 180-197
\end{tabular}

yecto de Mondragón: Cooperación (propietarios y protagonistas), Participación (compromiso en la gestión), Responsabilidad Social (distribución solidaria de la riqueza e implicación en el entorno) e Innovación (renovación permanente). Como muestra la siguiente cita, el grupo Mondragón plantea claramente la relevancia económica y social de la formación:

Las actividades del conocimiento y la divulgación de nuestra Experiencia Cooperativa pueden constituir un foco del que emane un contexto propicio para el desarrollo empresarial y social. La transmisión del saber hacer y los conocimientos acumulados, dentro y fuera de la Corporación, es una manera más de distribución solidaria de la riqueza. Las actividades del conocimiento y la divulgación de nuestra Experiencia Cooperativa pueden constituir un foco del que emane un contexto propicio para el desarrollo empresarial y social. La transmisión del saber hacer y los conocimientos acumulados, dentro y fuera de la Corporación, es una manera más de distribución solidaria de la riqueza. (Mondragón, 2007, 39).

\section{2.- DE LA REPRODUCCIÓN A LA TRANSFORMACIÓN}

\section{1.- Origen e impacto social del modelo de la reproducción}

Dentro de la sociología de la educación se ha denominado modelo de la reproducción al sostenido a partir de los años setenta por autores como Bourdieu y Passeron (1977), Bowles y Gintis (1985) o Baudelot y Establert (1976), desde el que se afirmaba que la función de la escuela era la reproducción de las desigualdades sociales. Althusser (1970) inició la aplicación de este modelo al análisis de la educación desde el estructuralismo marxista y, lejos de realizar un análisis científico y serio de la realidad social y de la obra de Marx, desprestigió las ciencias sociales. Escribió Para leer el Capital (Althusser y Balibar, 1969), una de las obras desde las que creó el estructuralismo marxista sin haber leído El Capital de Marx (1980), tal como reconoció él mismo más adelante (Althusser, 1995, 196-197).

Las aportaciones de Althusser tuvieron un fuerte efecto desmovilizador ya que, a pesar de denominarse "marxista”, favorecían la desactivación de reivindicaciones igualitarias. Así, por ejemplo, el profesorado, alumnado, familiares y movimientos sociales que buscaban la forma de superar las desigualdades sociales a través de la educación fueron catalogados de ilusos y no científicos. Se calificaba de inocencia humanista tanto el pensamiento de autores de gran reconocimiento internacional, Paulo Freire es un ejemplo, como las transformaciones igualitarias de los movimientos sociales progresistas, entre ellos las de los colectivos afroamericanos (Flecha y Serradell en Fdez. Palomares, 2003).

En este contexto tan adverso, el informe Jencks (1972) proporcionó la prueba empírica a una parte importante de sociólogos de la educación de la academia de los años 70 para defender que "la escuela no es responsable de las desigualdades sociales y no las cambia”. De hecho, éste fue el título con el que se tradujo dicho informe en España, en

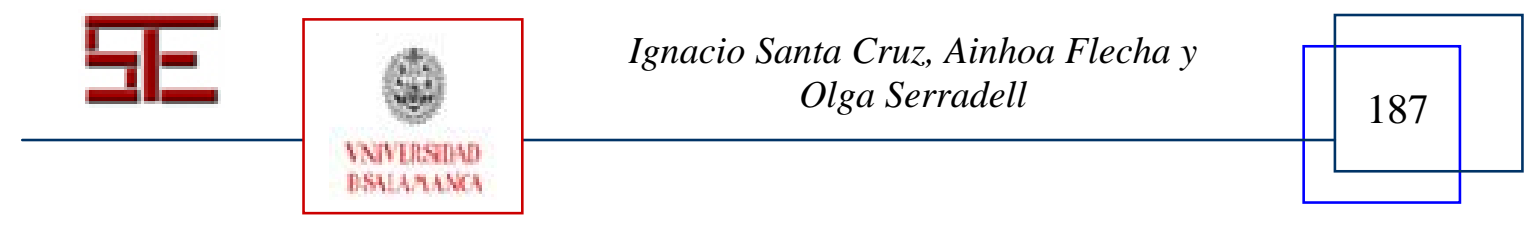


1985, años después de que su propio autor reconociera incorrecciones en los cálculos (Jencks, 1979). En aquel informe de 1972, Jencks consideraba inútil invertir esfuerzos en reformas educativas. Afirmaba que las diferencias entre escuelas tienen un efecto muy pequeño en lo que ocurre a los estudiantes después de la graduación y que una reforma escolar no tendría una influencia significativa en el grado de desigualdad entre las personas adultas (Bane y Jencks, 1972 41). Con estos datos, cualquier intento político de reformar la escuela quedaba desautorizado y desactivada cualquier iniciativa de la ciudadanía por reducir la desigualdad.

Más adelante, junto al propio Jencks, varios de los autores reproduccionistas se desvincularían de este modelo. Bourdieu, por ejemplo, no quiso que se le valorara por su obra La reproducción (Bourdieu y Passeron, 1977), la que él consideraba más estructuralista. De hecho, defendió una pedagogía que tenga en cuenta la desigualdad cultural socialmente condicionada y se oriente a reducirla. Tampoco Bowles y Gintis (1985) quisieron ser recordados por el modelo de la reproducción y se retractaron de él dirigiendo sus trabajos hacia el ámbito de la economía, donde han realizado importantes contribuciones.

Las teorías de la reproducción defienden que la educación no puede generar transformaciones, pero los autores que las defendieron en su momento sí creyeron en la posibilidad de transformar sus vidas, aumentar sus currículos y mejorar en la escala laboral y social. Sin embargo, los importantes problemas éticos que plantea este modelo no son su única crítica, sino también su falta de rigor científico. Por un lado, no existe un impacto de los resultados de estas teorías en la mejora de las condiciones de vida de la ciudadanía, por lo que podemos afirmar que sus análisis y conclusiones no tienen ninguna utilidad social. Por otro, su influencia fue doblemente contraproducente ya que no solamente desprestigió teorías científicas basadas en el análisis de las realidades transformadoras, sino que también influyó negativamente a iniciativas que buscaban mejorar las condiciones de vida de las personas a través de la educación.

En los años 50 y 60 el movimiento por los derechos civiles en Estados Unidos empezaba a transformar la sociedad en busca de la igualdad entre afroamericanos y blancos. Al mismo tiempo, desde la academia, el estructuralismo marxista (Althusser, 1995; Althusser y Balibar, 1969) ponía frenos a esa transformación negando la capacidad de los sujetos para superar las desigualdades sociales y educativas. Quienes defendían la igualdad a través de la práctica, ya fuera desde una implicación política o desde la participación en experiencias educativas transformadoras, creían en la posibilidad de cambio. El modelo de la reproducción se alejaba de estas alternativas a la vez que desmovilizaba. El actual presidente de Estados Unidos es afroamericano, un hecho real que acerca la sociedad estadounidense al sueño de igualdad de Martin Luther King y del movimiento por los derechos civiles. La contribución de las teorías de la reproducción a esta igualdad ha sido y sigue siendo nula.

Como hemos visto en la primera parte del artículo, experiencias como Mondragón demuestran que la educación puede ser transformadora y mejorar las condiciones de vida

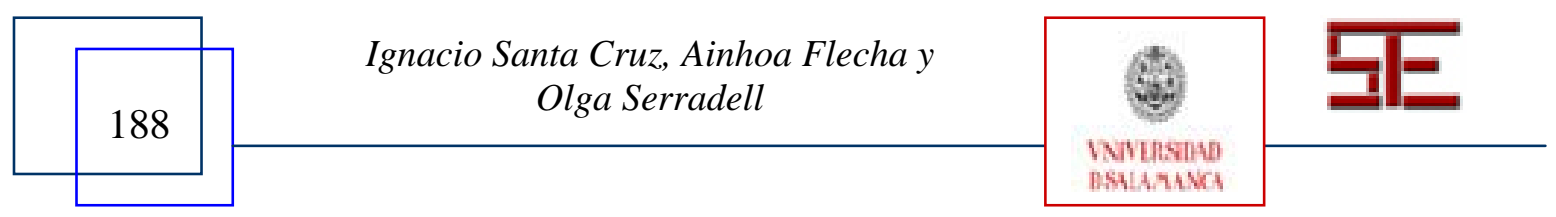




\begin{tabular}{c|c}
\hline 1 & $\begin{array}{c}\text { Revista Electrónica Teoría de la Educación. } \\
\text { Educación y Cultura en la Sociedad de la Información. }\end{array}$ \\
\hline & TESI, 10(3), 2009, 180-197
\end{tabular}

de las personas. Siguiendo con el análisis de la influencia e impacto de las teorías en la reproducción o transformación de la desigualdad en nuestras sociedades, en el apartado siguiente profundizamos sobre la orientación y utilidad social de las teorías sociológicas y de la educación de mayor relevancia científica internacional.

\section{2.- La educación como transformación}

Podemos decir que las contradicciones que inundan el modelo reproduccionista tienen dos bases: a) la debilidad teórica de los autores que lo plantean, fruto básicamente de la falta de lectura, reflexión y discusión en torno a las obras más importantes de las ciencias sociales y de la educación, y b) la falta de vinculación de estos autores con movimientos sociales igualitarios y prácticas transformadoras. Después de una época de hegemonía estructuralista en el ámbito académico, desde hace unos años se está dando lo que algunos autores han definido como el giro dialógico de las ciencias sociales (Flecha, Gómez y Puigvert, 2001), dentro del que se enmarcan las contribuciones teóricas de los autores más relevantes en ciencias sociales, como Ulrich Beck, Jon Elster, Paulo Freire, Anthony Giddens, Jürgen Habermas o Alain Touraine. Las teorías sociológicas más importantes de la actualidad se orientan a la transformación y realizan una sociología rigurosa, tanto a nivel intelectual como científico que es útil para los ciudadanos y ciudadanas. En esta línea se encuentran también autores de gran prestigio internacional tales como Erik Olin Wright (Fung y Wright, 2003) o Michael Burawoy (2009), quienes apuestan por una sociología de calidad, con fuertes bases científicas, y que esté alejada del elitismo académico de Althusser, es decir, que sea de interés para la sociedad.

Este giro dialógico también se da en educación, donde el modelo de la reproducción está siendo desbancado por un modelo orientado a la transformación social en el que la educación juega un papel muy importante. Entre los autores que se sitúan en esta tendencia se encuentran Basil Bernstein, Michael Apple, Paul Willis o Henry A. Giroux. Bernstein (1990) desarrolló una teoría del discurso pedagógico en la que distinguía entre lo transmitido, único aspecto tenido en cuenta por el modelo de la reproducción, y la transmisión, elemento clave para entender qué ocurre en los procesos de aprendizaje en la escuela. Apple introdujo en el análisis sociológico algo que el modelo de la reproducción no contemplaba, a saber, el estudio de las relaciones de poder externas e internas a la escuela y de los modelos de resistencia al poder, las luchas de los trabajadores, los movimientos de mujeres y los grupos culturales (Apple y Beane, 1997). Willis (1994) planteó la escuela como un contexto de producción cultural e ideológica y señaló la importancia de las interacciones que se dan entre los sujetos durante este proceso productivo. Definir los contextos educativos como productores de cultura distinguía su perspectiva de cualquier reproduccionismo teórico. Finalmente y de forma más global, Giroux (2001) planteó la educación como un proyecto político y desde una perspectiva transformadora. Este autor otorgaba a los sujetos la capacidad de transformar la realidad, y al profesorado un papel fundamental, el de intelectuales transformativos.

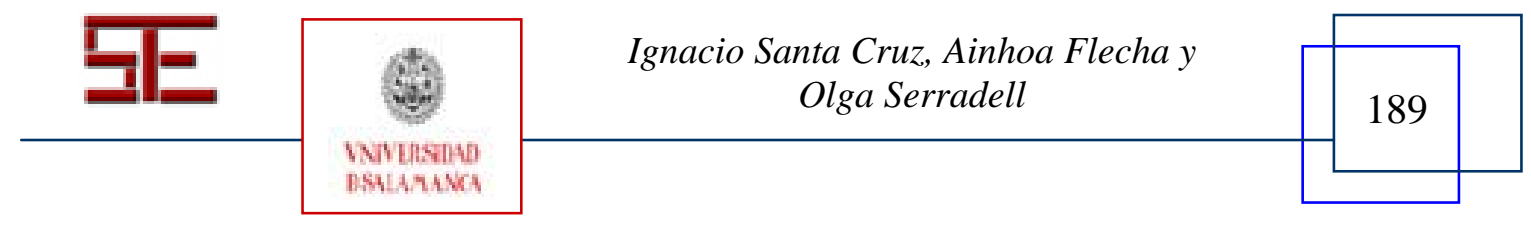


Lo que diferencia las teorías de la educación reproduccionistas de las que se orientan a la transformación es que mientras las primeras invalidan la utilidad de la educación para superar las desigualdades sociales, las segundas analizan, no sólo cómo se reproducen estas desigualdades, sino también cómo funcionan las actuaciones, prácticas y experiencias que contribuyen a su superación. En esta línea ya se han posicionado organismos internacionales como la Unión Europea. Con la finalidad de clarificar cuáles son las actuaciones que están generando éxito educativo y cuáles fracaso en los Estados y sociedades europeas actuales y aplicar en sus centros educativos las actuaciones que aconseja la comunidad científica internacional en educación, la UE decidió por primera vez dedicar uno de sus proyectos de investigación de primer nivel a la educación escolar, el proyecto integrado del VI Programa Marco de la Comisión Europea INCLUD-ED. Strategies for inclusion and social cohesion in Europe from education (CREA-UB, 2006-2011).

Las teorías sociológicas de la educación actuales de mayor reconocimiento científico superan los dos problemas básicos del enfoque reproduccionista, por un lado la debilidad teórica, ya que se basan en la lectura en profundidad y en el análisis riguroso de las obras más importantes de las ciencias sociales y de la educación. Por el otro, incluyen en sus análisis las propuestas de los movimientos sociales igualitarios y las aportaciones de los agentes sociales a los que van dirigidas las investigaciones y estudios realizados desde la academia. Este último es uno de los elementos más importantes para conseguir que los resultados de las investigaciones y teorías desarrolladas tengan una utilidad social, es decir, proporcionen los elementos de análisis necesarios que permitan a los sujetos reflexionar sobre sus situaciones y llevar a cabo actuaciones individuales y colectivas que mejoren sus condiciones de vida.

Como ya hemos visto, la inversión en formación ha constituido parte de la estrategia empresarial de Mondragón desde su inicio. La educación es, pues, uno de los elementos clave que caracterizan y explican el éxito de este proyecto. La inversión educativa se ha ampliado a lo largo de los años adaptándose y modificando su oferta para dar respuesta a las nuevas demandas y necesidades que plantea la sociedad de la información. Los Centros de Formación Profesional, los Centros para la Formación Continua y la Universidad de Mondragón forman parte de la estructura del grupo empresarial y cubren necesidades formativas, no solamente de las cooperativas, sino también de la sociedad en general. Formación, capital humano, desarrollo tecnológico y relación con empresas y promoción empresarial son actividades que se encuentran todas ellas integradas en los proyectos de los centros formativos de Mondragón.

Además, de las cuatro áreas que configuran Mondragón: Finanzas, Industria, Distribución y Conocimiento, esta última incluye la formación como uno de sus aspectos más importantes, junto a la investigación y la innovación tecnológica. Así, la variable "educación” es parte integrante de la estrategia empresarial de Mondragón, como elemento de desarrollo que proporciona mayor competitividad y da continuidad al proyecto. Ejemplo de ello son el importante crecimiento de la MU en menos de 10 años en lo que a titulaciones, alumnado e investigación se refiere, así como las actividades de forma-

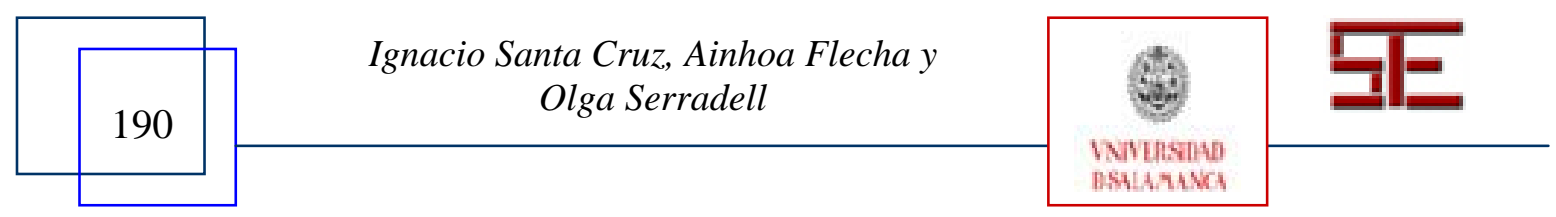




\begin{tabular}{c|c}
\hline 1 & $\begin{array}{c}\text { Revista Electrónica Teoría de la Educación. } \\
\text { Educación y Cultura en la Sociedad de la Información. }\end{array}$ \\
\hline & TESI, 10(3), 2009, 180-197
\end{tabular}

ción para las y los trabajadores en activo y el trabajo de inserción laboral realizado en los centros formativos.

En concreto, el desarrollo de un modelo educativo propio, Mendeberri, para dar respuesta a los nuevos retos de la sociedad y, en concreto, a las necesidades surgidas en las empresas e instituciones, es clave para entender cómo desde Mondragón se crea una oferta educativa que responde a las demandas de su entorno, así como a las de la sociedad actual. Ello implica un cambio en la forma de entender la educación, pasando de entender el proceso de enseñanza como transmisión de conocimientos a desarrollar una formación que proporciona a las personas las habilidades necesarias para enfrentarse a los nuevos retos laborales de la sociedad de la información. Por esa razón Mendebarri, además de ser un modelo trilingüe (euskera, castellano e inglés), incluye tres dimensiones: formación personal, social y profesional. Por consiguiente, los alumnos y alumnas son considerados agentes proactivos, con capacidad de participación, colaboración y asunción de responsabilidades.

Este modelo se puso en marcha en el curso 2002-2003 a raíz del trabajo que desde 1999 se venía realizando desde la MU, y en el curso 2006-2007 ya lo aplicaban el 70\% de las titulaciones. Uno de los aspectos más importantes a destacar es que el proceso de diseño de Mendeberri es el resultado de la participación y colaboración entre diferentes agentes, empresas, centros de educación, grupo de expertos, profesores y ex alumnos de áreas de conocimiento relacionados con las titulaciones. Este procedimiento basado en el análisis riguroso de la situación, el diálogo entre diferentes agentes sociales y la búsqueda de acuerdos tuvo como resultado la creación del "perfil profesional” que reúne, entre otros aspectos, aquellas aptitudes que el alumnado debe desarrollar, a saber, aptitudes técnicas, metodológicas, personales, sociales e incluso transversales que permitan identificar a aquellos profesionales formados en su comunidad. Este perfil implica un amplio consenso social para cada titulación que, además, está totalmente acorde con los criterios del Espacio Europeo de Educación Superior (EEES). Actualmente 168 profesoras y profesores de la MU siguen trabajando en el diseño, desarrollo y aplicación de este nuevo modelo educativo.

Por un lado, Mondragón muestra las consecuencias positivas empresariales y sociales de incorporar en su estructura una concepción dialógica de la educación y que se encuentra en perfecta sintonía con el trabajo desarrollado por los autores de mayor relevancia científica internacional, y en concreto Paulo Freire (1995). Por otro lado, esta experiencia falsea las teorías de la reproducción. Además de ser competitiva e integrarse perfectamente en la economía informacional, ha obtenido mejores condiciones laborales y sociales para los trabajadores y trabajadoras que otras empresas capitalistas. Hace explícita la aplicación de una "ética en los negocios” basada en el respeto a los Derechos Humanos, el cumplimiento de las leyes y normas locales vigentes o la no discriminación en los procesos de contratación, entre otros.

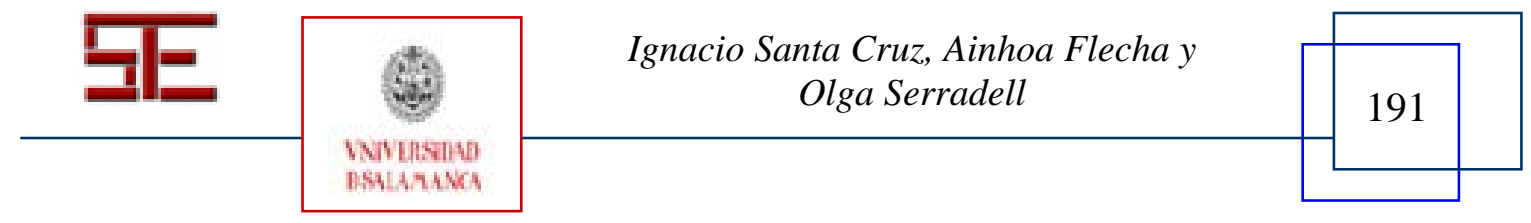




\section{3.- EL ÉXITO ECONÓMICO Y SOCIAL DE UNA EXPERIENCIA SOLIDARIA}

El camino seguido por las cooperativas del grupo Mondragón desde 1956 hasta la actualidad refleja el éxito económico y social de esta experiencia. Como pone de manifiesto su Informe Anual de 2007, Mondragón es a finales de ese año "el primer grupo empresarial vasco y el séptimo de España”. A partir de algunos datos de ese año se obtiene una aproximación de la dimensión adquirida por el grupo empresarial de Mondragón que mostramos en la siguiente tabla:

Tabla 1. Magnitudes relevantes del grupo Mondragón a 31 de diciembre de 2007

\begin{tabular}{|l|l|}
\hline ACTIVOS TOTALES & $\mathbf{3 2 . 8 4 0}$ \\
\hline INGRESOS & 16.377 \\
\hline VENTAS & 15.056 \\
\hline RESULTADOS & 792 \\
\hline PLANTILLA & 103.731 \\
\hline
\end{tabular}

Fuente: Elaboración propia a partir de los datos del Informe Anual 2007 (Mondragón, 2008). (Todos los datos en millones de euros, excepto plantilla en unidades).

En la actualidad el grupo Mondragón está estructurado en cuatro áreas: Finanzas, Industria, Distribución y Conocimiento. Destaca la presencia internacional del grupo, orientado a competir en los mercados globales, y puesta de manifiesto especialmente en el área industrial, de la cual son indicativos los datos que se exponen a continuación.

En primer lugar, en el año 2007, de las ventas totales del área industrial (7.439 millones de euros) un 56,9\% se realizaron a nivel internacional. En ese año el grupo tenía 75 establecimientos en el extranjero de los cuales 6 son delegaciones corporativas y 69 son filiales productivas. Además, el grupo Mondragón está presente en 17 países mediante filiales productivas, 9 países de la Unión Europea (Alemania, Polonia, Reino Unido, Chequia, Eslovaquia, Francia, Italia, Portugal y Rumania) y otros 8 de distintas zonas del mundo (China, India, Tailandia, Brasil, México, Estados Unidos, Marruecos, Sudáfrica y Rusia). Además, en 2007 el volumen del empleo internacional ascendió a 16.580 personas. Este dato representa el 16\% del total del empleo del grupo (103.731 personas). Pero otro de los datos que pone de manifiesto el éxito, tanto económico como social, del grupo Mondragón es la evolución del empleo que en los últimos 20 años ha pasado de 20.818 personas en el año 1987 a 103.731 en el 2007.

Los datos anteriores ponen de manifiesto el éxito conseguido por la experiencia de Mondragón que, partiendo de ser una pequeña cooperativa fundada en 1956 por un profesor de formación profesional y 5 alumnos, es actualmente el primer grupo empresarial vasco y el séptimo español. Pero, además, presenta un modelo de cooperativismo competitivo a nivel global que tiene una presencia muy importante en los mercados internacionales.

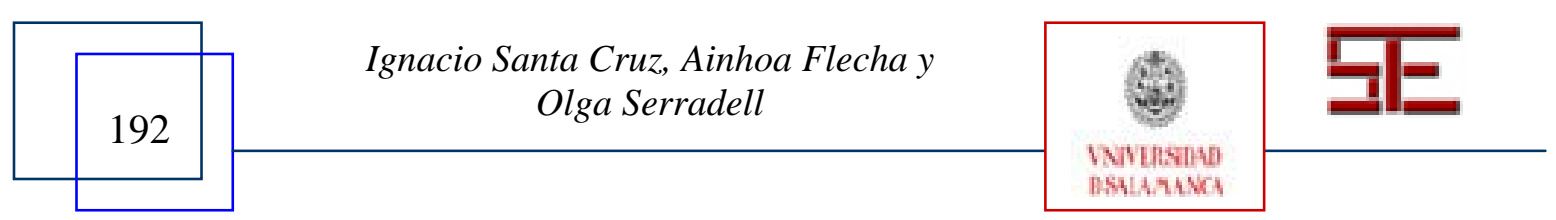




\begin{tabular}{c|c}
\hline 1 & $\begin{array}{c}\text { Revista Electrónica Teoría de la Educación. } \\
\text { Educación y Cultura en la Sociedad de la Información. }\end{array}$ \\
\hline & TESI, 10(3), 2009, 180-197
\end{tabular}

Un segundo aspecto a destacar de la experiencia de Mondragón es la creación de más y mejores empleos a lo largo de toda su trayectoria, empleos que se han mantenido en los momentos de las crisis económicas más importantes que se han dado en las últimas décadas como las de 1973 y 1993. La zona donde nació el grupo Mondragón y en la que tiene mayor influencia, es donde existen menores desigualdades en la distribución de la renta per cápita de España. Esto es porque el grupo tiene unas políticas de remuneración de sus miembros que acotan las diferencias salariales en las empresas y por lo tanto limitan las desigualdades. Así, por ejemplo, los beneficios generados por las inversiones que realizan en el exterior son reinvertidos en el país donde se generan y las remuneraciones a los trabajadores que contratan en estos países tienen que estar como mínimo en un $10 \%$ por encima del salario mínimo de esas zonas.

Las crisis económicas crean importantes problemas a los trabajadores, a los Estados y a las empresas. Con ellas se produce una importante pérdida de puestos de trabajo que afecta a muchos trabajadores y trabajadoras. Otros, ante el riesgo de pérdida de empleo, aceptan condiciones de trabajo que suponen una pérdida de derechos, de calidad de trabajo y de vida. En lo que a los Estados se refiere, éstos se enfrentan a problemas presupuestarios para hacer frente a los cuantiosos gastos que generan las medidas necesarias para actuar ante la crisis, al mismo tiempo que se producen procesos de pérdida de legitimidad ante el conjunto de la población. Finalmente, muchas empresas se ven empujadas al cierre o a paralizar sus planes de crecimiento. Todos estos procesos hacen que aumenten los riesgos de exclusión social para el conjunto de la sociedad, pero también que disminuya la solidaridad y la cohesión social entre sus miembros.

La crisis actual está generando un debate sobre qué medidas tomar para hacer frente a la misma. Desde una postura se defiende dejar al mercado que resuelva los problemas a su forma, eliminando las empresas y los sectores no rentables. Desde esta perspectiva se considera que ésta es la forma de asegurar la creación de un punto de partida sano y fuerte que dé paso a una fase expansiva del ciclo con fundamentos sólidos. Sin embargo, desde la aproximación mayoritaria en este momento, la salida de la crisis pasa por una intervención del Estado en la economía y por un incremento de la regulación y control de la misma. Dentro de la misma postura se dan diferentes tendencias que discrepan sobre la duración y la forma de la intervención, tales como si deben hacerse o no nacionalizaciones. Pero quienes defienden esta postura coinciden en la necesidad de una regulación más estricta, especialmente en lo relativo a los mercados financieros.

Mondragón presenta una alternativa no capitalista de organización empresarial que da respuesta a algunos de los problemas planteados por las crisis económicas. En relación al problema del paro, cuando una empresa del grupo tiene problemas o entra en crisis, otras le ayudan financieramente o emplean a los trabajadores que no tienen trabajo en la empresa en crisis. De esta forma, no solamente se evita la destrucción de empleos en el conjunto de las empresas, sino que además tiene un efecto positivo sobre las zonas donde éstas están ubicadas. Éste no es el único mecanismo de solidaridad entre las empresas del grupo, existen otros que toman diversas formas. Están, por ejemplo, la Reconversión de Resultados o el Fondo de Solidaridad Corporativo (FSC). El primero consis-

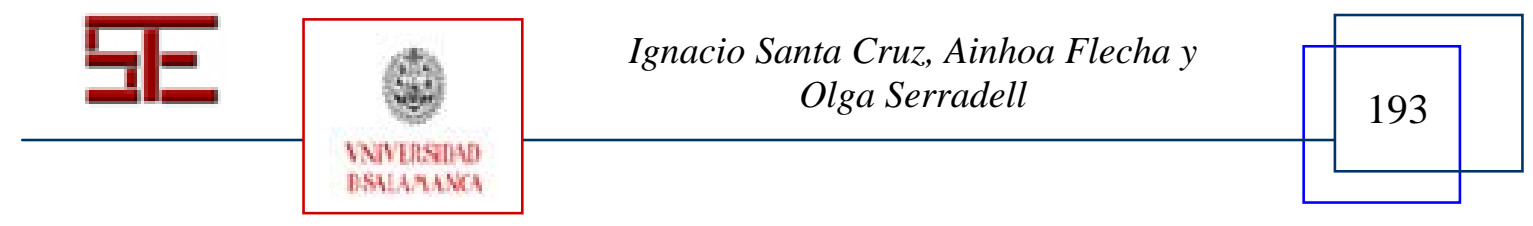


tente en que cooperativas del grupo con beneficios transfieran parte de éstos a otras cooperativas menos beneficiadas; el FSC es un sistema creado en el año 2003 y que tiene como finalidad complementar la Reconversión de Resultados y apoyar a las cooperativas en pérdidas.

Otro aspecto importante que aporta la experiencia de Mondragón es la reducción de desigualdades gracias a la existencia de unos abanicos salariales limitados. Ciertamente, existen otras experiencias cooperativas que tienen abanicos más igualitarios que los que presenta Mondragón, pero lo que caracteriza a esta experiencia es que compite con éxito en los mercados globales sin renunciar a disminuir las desigualdades. Así, por ejemplo, en el año 2007 las remuneraciones de un 97\% de los socios cooperativistas se situaban en el abanico salarial de 1 a 3,49. De esta forma, sólo un 3\% de los socios quedaba por encima del 3,50\% y un 85\% se situaba en el abanico de 1 a 2,5 (Mondragón, 2008, 64). Si se compara este dato con las diferencias salariales existentes en las empresas con las que compite el grupo Mondragón en los mercados globales, se ve claramente que esta experiencia empresarial representa un modelo para la reducción de desigualdades en las sociedades actuales.

Otro aspecto a destacar es el funcionamiento democrático de todas las empresas y del grupo Mondragón. Las cooperativas en general tienen una forma de funcionamiento y gestión basada en mecanismos democráticos de participación a todos los niveles de la empresa, lo que destaca en Mondragón es que la forma de funcionamiento democrático se da en un grupo que compite en los mercados internacionales. Este planteamiento queda claramente reflejado en su misión que es definida por Mondragón de la siguiente forma:

La Misión de Mondragón aglutina los objetivos básicos de una organización empresarial que compite en los mercados internacionales con la utilización de métodos democráticos en su organización societaria, la creación de empleo, la promoción humana y profesional de sus trabajadores y el compromiso de desarrollo con su entorno social (Mondragón, 2008, 54).

\section{4.- CONCLUSIONES}

El grupo Mondragón ha conseguido un importante éxito empresarial pero también social, en el sentido de reducción de las desigualdades y de la superación de tres crisis económicas. Este hecho no puede desvincularse de los principios básicos y valores corporativos que desde su inicio han orientado la experiencia y que se concretan en tres ámbitos de actuación: Educación Cooperativa, Participación-Cooperación y Transformación Social.

La importancia que esta experiencia da a la formación a lo largo de su historia refuta las teorías de la reproducción en educación. Este artículo muestra que la formación es desde sus orígenes hasta la actualidad uno de los pilares del grupo Mondragón, de su éxito empresarial y de su relevancia internacional. Pero la trayectoria de Mondragón invalida

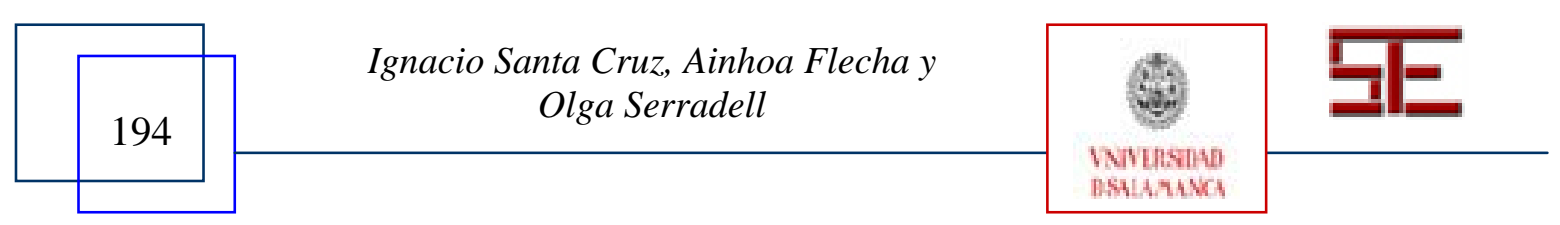




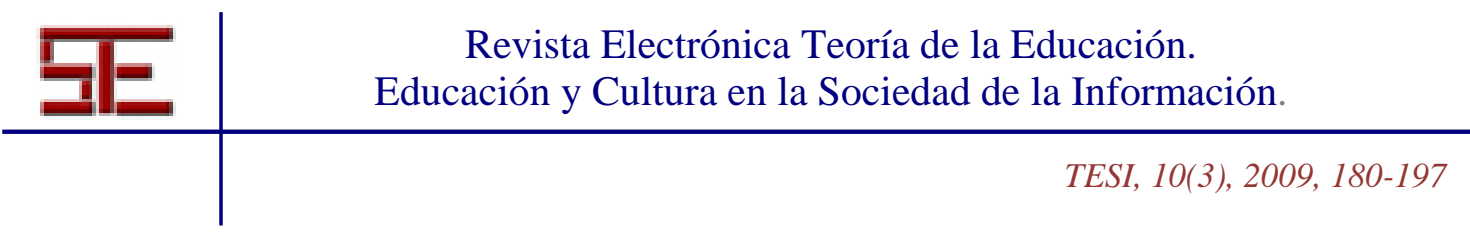

también cualquier discurso reproduccionista, es decir, cualquier otra teoría que plantee la imposibilidad de la transformación social.

Los análisis científicos de la sociedad requieren tanto de teorías como del estudio de realidades empíricas que permitan contrastarlas, aportar alternativas y nuevos elementos de utilidad para superar las situaciones de desigualdad. Éste ha sido y sigue siendo en la actualidad el papel de la educación en la experiencia de Mondragón, donde la formación ha constituido un elemento de mejora y de transformación tanto individual como colectiva.

Experiencias como ésta pueden contribuir enormemente a la elaboración de nuevas políticas económicas de ámbito nacional e internacional. Mondragón está consiguiendo mantener la calidad y cantidad de los puestos de trabajo en la actual situación de crisis económica, de la misma forma que lo hizo en las anteriores de 1973 y 1993. Pero además, todas las personas, también aquellas que forman parte de grupos socialmente vulnerables como jóvenes, mujeres o personas con bajos niveles educativos, acceden a la mejora de la calidad de los trabajos y de las condiciones de vida. La organización empresarial del grupo Mondragón está basada en principios no capitalistas entre los que se encuentran la solidaridad y la mejora de las condiciones de vida de las personas. Es en ese sentido que experiencias como la de Mondragón son tan positivas, ya que desarrollan nuevas formas de organización del trabajo que consiguen combinar productividad y satisfacción de los trabajadores y trabajadoras. El origen de su alto nivel de competitividad se encuentra en la propia implicación de los trabajadores en las compañías del grupo empresarial y en las redes de solidaridad que éstos han creado entre las cooperativas que forman el grupo.

Así, contrariamente a lo que plantean las teorías de la reproducción, Mondragón demuestra que es posible contribuir a la superación de las desigualdades sociales, no únicamente en el ámbito educativo, sino como alternativa global.

\section{5.- BIBLIOGRAFÍA}

ALTHUSSER, L. (1970): Ideologie et appareils ideologiques d'Etat, La Pensee, 151, París.

-- (1995): El porvenir es largo. Barcelona: Destinolibro (p.o. 1992).

ALTHUSSER, L. y Balibar, E. (1969): Para leer el capital. México, Siglo XXI (p.o. 1967).

APPLE, M. y BEANE, J.A. (1997): Escuelas democráticas. Madrid, Morata (p.o. 1997).

AUBERT, A.; FLECHA, A.; GARCÍA, C.; FLECHA, R. Y RACIONERO, S. (2008): Aprendizaje Dialógico en la Sociedad de la Información. Barcelona, Hipatia.

BAUDELOT, C. H. y ESTABLET, R. (1976): La educación capitalista en Francia. Madrid, S.XXI.

BERNSTEIN, B. (1990): Poder, educación y conciencia. Sociología de la transmisión cultural. Barcelona, El Roure.

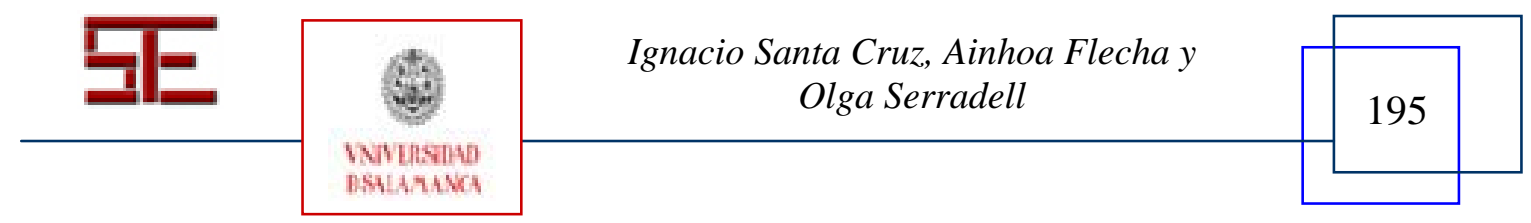


BOURDIEU, P. y PASSERON, J. C. (1977) La Reproducción. Elementos para una teoría del sistema de enseñanza. Barcelona, Laia.

BOWLES, S. y GINTIS, H. (1985): La instrucción escolar en la América capitalista. Madrid, S. XXI.

BURAWOY, M. (2009): The Extended Case Method: Four Countries, Four Decades, Four Great Transformations, and One Theoretical Tradition. University of California Press.

COMMISSION OF THE EUROPEAN COMMUNITIES (2005): Communication to the spring European council. Working together for growth and jobs. A new start for the Lisbon Strategy. Brussels, European Commission.

CREA-UB (2006-2011): INCLUD-ED. Strategies for inclusion and social cohesion in Europe from education, proyecto integrado del VI Programa Marco de la Comisión Europea.

EUROPEAN COUNCIL (2000): Presidency Conclusions. Lisbon European Council (Lisbon, EC).

FLECHA, R.; GÓMEZ, J. y PUIGVERT, L. (2001): Teoría Sociológica Contemporánea. Madrid, Paidós.

FLECHA, R. y SERRADELL, O. (2003): Capítulo III. El desarrollo de la sociología de la educación. Principales enfoques o escuelas. Revisión crítica, en Fdez. Palomares, : Sociología de la Educación. Madrid, Pearson Educación.

FREIRE, P. (1995): Pedagogía del oprimido. Madrid, Siglo XXI (p.o. 1969).

FUNG, A. Y WRIGHT, E. O. (2003): Deepening Democracy: Institutional Innovations in Empowered Participatory Governance. The Real Utopias project. LondonNew York, Verso.

GIROUX, H. (2001): Cultura, política y práctica educativa. Barcelona, Graó (p.o. 2000).

JENCKS, C. (1972): Inequality: A Reassessment of the Effect of Family and Schooling in America. Nueva York-Londres, Basic Books.

-- (1973): Inequality in retrospect, Harvard Educational Review, 43 (1), 138-164.

-- (1979): Who Gets Ahead? The Determinants of Economic Success in America. New York, Basic Books.

JENCKS, C. y BANE, M. J. (1985): La escuela no es responsable de las desigualdades sociales y no las cambia, en Gras, A. Sociología de la educación. Textos fundamentales. Madrid, Narcea.

MARX, K. (1980): El Capital. Crítica de la economía política. Madrid, Siglo XXI (p.o. 1867, 1885, 1894).

MONDRAGÓN (2007): Historia de Una Experiencia. Accesible desde la página web: http://www.mcc.es/esp/quienessomos/historiamcc_esp.pdf .

-- (2008): Informe Anual 2007. Mondragón: Litografía Dannona. Accesible desde la página web: www.mcc.es

WILLIS, P. (1988): Aprendiendo a trabajar. Madrid, Akal (p.o. 1977).

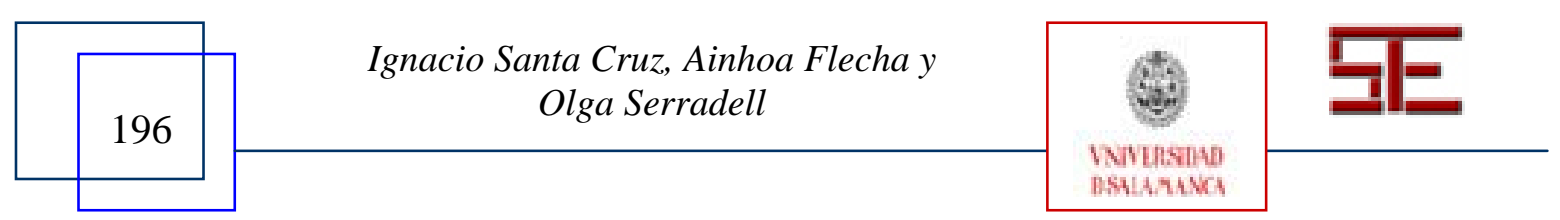




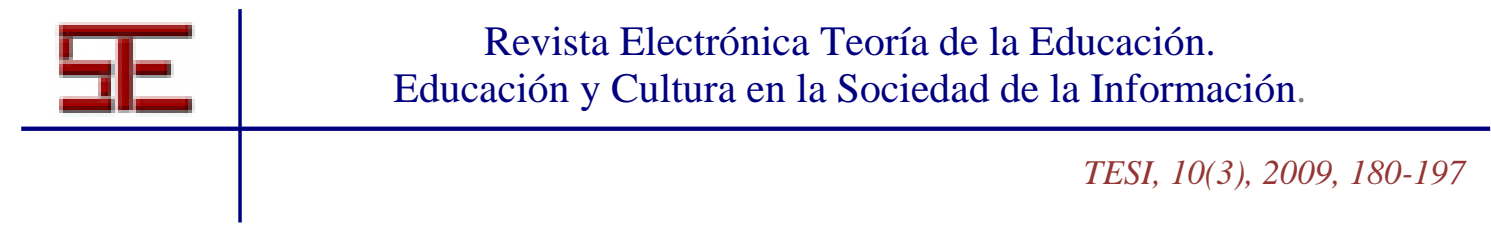

Para citar el presente artículo puede utilizar la siguiente referencia:

SANTA CRUZ, I., FLECHA, A. y SERRADELL, O.: (2009): La promoción de una economía no-capitalista: el Grupo Mondragón, en FLECHA GARCÍA, R. y STEINBERG, S. (Coord.) Pedagogía Crítica del S.XXI [monográfico en línea]. Revista Electrónica Teoría de la Educación: Educación y Cultura en la Sociedad de la Información. Vol. 10, $\mathrm{n}^{0}$ 3. Universidad de Salamanca, pp. 180-197. [Fecha de consulta: $\mathrm{dd} / \mathrm{mm} /$ aaaa $]$.

http://campus.usal.es/ revistas_trabajo/index.php/revistatesi/article/view/3969/3991

ISSN: $1138-9737$

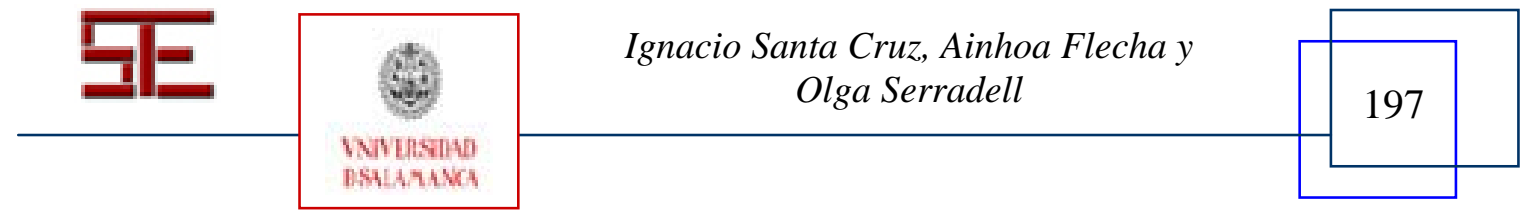

